\title{
Appearances can be deceiving: What is the diagnosis for this community-acquired pneumonia?
}

\author{
Frank YH Lin $\mathrm{MD}^{1}$, Coleman Rotstein MD FRCPC ${ }^{1,2}$
}

\begin{abstract}
A 65-year-old male with a past history of cigarette smoking and asbestos exposure (in the 1970s) presented to the emergency department with a one-month history of progressive dyspnea, right-sided pleuritic chest pain, cough productive of white-coloured sputum and malaise. His health problems had commenced four months before presentation while he was vacationing at a northern Ontario resort. At that time, he had felt unwell and had developed a fever with rightsided pleuritic chest pain that radiated to his right shoulder. The diagnosis was an upper respiratory tract infection, made by the local physician; the patient was treated with a 10-day course of cephalexin. Although his condition had initially improved after the antibiotic therapy, during the month before presentation he had experienced increasing fatigue, cough with clear sputum production and a loss of appetite. He also developed worsening right-sided pleuritic chest pain that radiated to the right shoulder, dyspnea and orthopnea. He had no nausea, vomiting, diarrhea or hemoptysis. However, he had lost $4 \mathrm{~kg}$ and had drenching night sweats over the previous three and a half months. Further history revealed that he had drunk well water during his vacation in northern Ontario and that several families who were with him at that time also became ill, although he was not aware of the nature of their symptoms.
\end{abstract}

On examination, he was a thin male in some respiratory distress and was afebrile. His blood pressure was $140 / 90 \mathrm{mmHg}$, and he had a heart rate of 80 beats/min and a respiratory rate of $30 \mathrm{breaths} / \mathrm{min}$. Chest examination revealed percussion dullness, decreased breath sounds, crackles, and bronchial breathing in the right base and mid-lung fields. No clubbing was observed. There was no jugular venous distention, and heart sounds were normal without any murmurs. His abdomen was flat, but he had mild right upper quadrant tenderness on deep palpation. No abdominal masses were palpable, no organomegaly was appreciated and there were no stigmata of chronic liver disease. Bowel sounds were present.

The patient's oxygen saturation was $89 \%$ on room air. The white blood cell count revealed mild leukocytosis of $12.2 \times 10^{9} / \mathrm{L}$ without a left shift, but hemoglobin and platelet counts were normal. The erythrocyte sedimentation rate was $38 \mathrm{~mm} / \mathrm{h}$. The level of serum aspartate aminotransferase was $28 \mathrm{U} / \mathrm{L}$, while the alanine aminotransferase level was mildly elevated at $47 \mathrm{U} / \mathrm{L}$; the alkaline phosphatase and gammaglutamyl transpeptidase levels were moderately elevated at $380 \mathrm{U} / \mathrm{L}$ and $243 \mathrm{U} / \mathrm{L}$, respectively. The patient's total bilirubin concentration was normal, but the albumin concentration was depressed at $32 \mathrm{~g} / \mathrm{L}$. All other serum chemistry values were within normal limits.

\footnotetext{
${ }^{1}$ Department of Medicine and ${ }^{2}$ Division of Infectious Diseases, McMaster University, Henderson Site, Hamilton Health Sciences Corporation, Hamilton, Ontario

Correspondence and reprints: Dr Coleman Rotstein, McMaster Medical Unit, Henderson Site, Hamilton Health Sciences Corporation, 711 Concession Street, Hamilton, Ontario L8V 1C3. Telephone 905-574-3301, fax 905-575-7320, e-mail crotstei@fhs.mcmaster.ca
} 


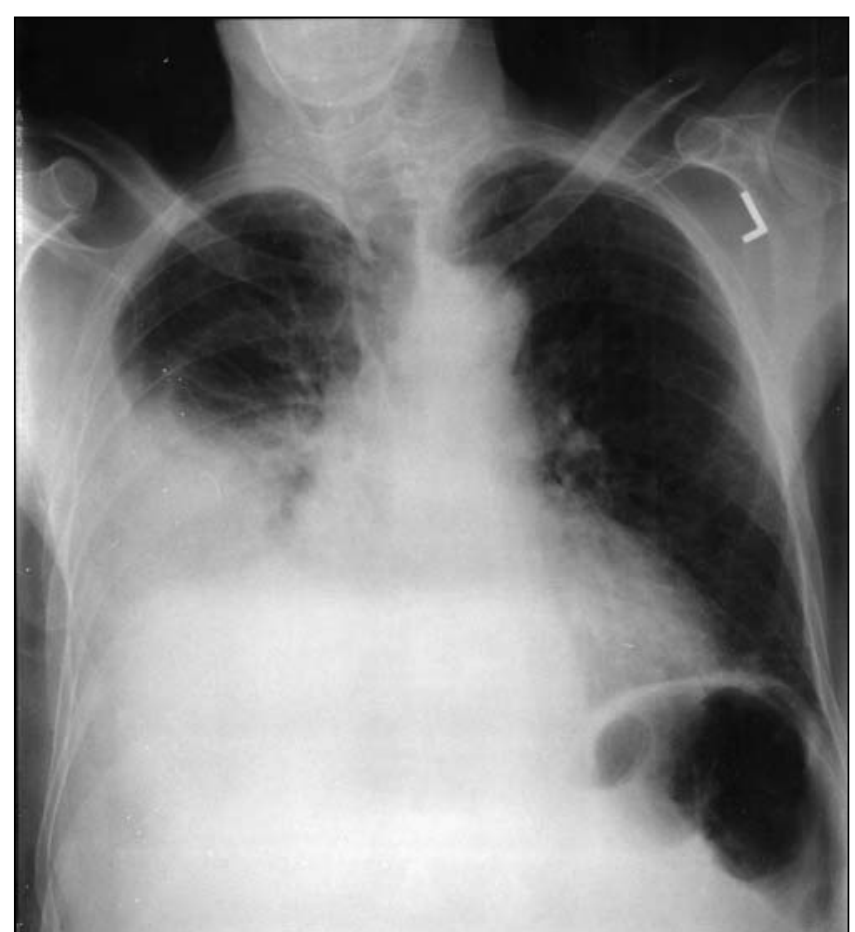

Figure 1) Chest radiograph demonstrating right middle and lower lobe consolidations with effusion

A chest X-ray (Figure 1) showed a large right pleural effusion with associated consolidation or subsegmental atelectasis in the rght middle and lower lung zones. An underlying parenchymal mass could not be excluded. Computed tomography of the thorax with contrast enhancement (Figure 2) revealed no masses in the lung parenchyma or the mediastinum. However, it demonstrated a $10 \mathrm{~cm}$ fluid collection occupying most of the right lobe of the liver, with peripheral enhancement consistent with a massive liver abscess.

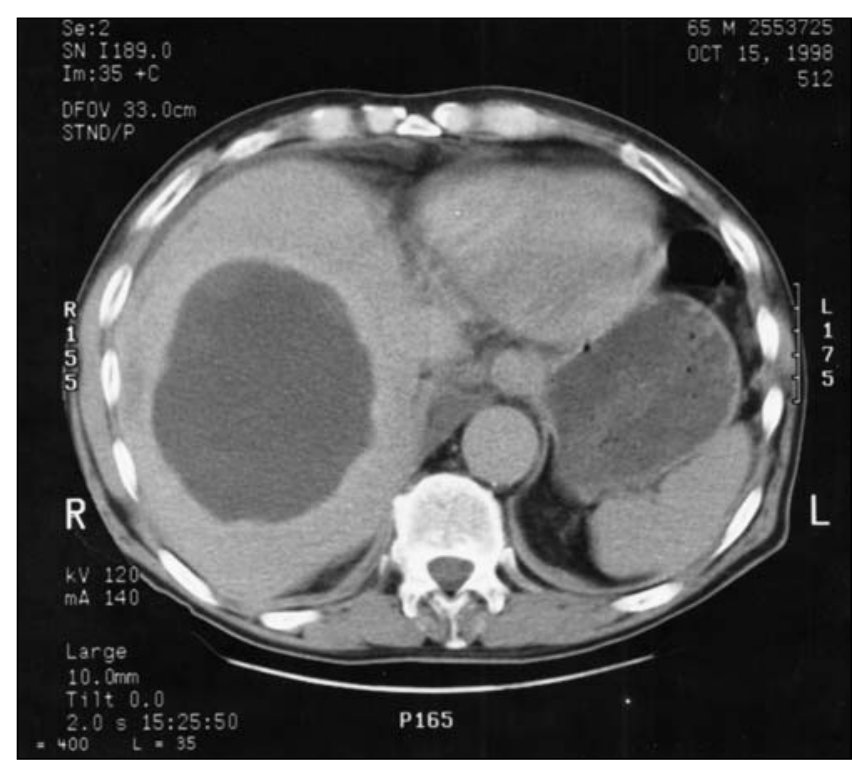

Figure 2) Computed tomography scan of the abdomen showing massive liver abscess in the right lobe of the liver

Percutaneous computed tomography-guided drainage of the liver abscess ensued, and $200 \mathrm{~mL}$ of bloody purulent fluid was aspirated. In addition, a chest tube was inserted, and $900 \mathrm{~mL}$ of fluid of similar consistency with numerous leukocytes was removed from the thorax. Microscopic examination of the fluids did not reveal any microorganisms, and cultures of the fluids and blood were negative for bacteria. Thereafter, therapy consisting of intravenous clindamycin $600 \mathrm{mg}$ every $8 \mathrm{~h}$ and oral ciprofloxacin $750 \mathrm{mg}$ bid was initiated.

What is your diagnosis? 
Continued from page 46

\section{DIAGNOSIS}

Entamoeba histolytica serology revealed a titre of $1: 12,800$ by immunoglobulin G-specific ELISA. However, examination of the stool did not reveal any eggs or parasites. On receipt of the serology report, the patient's treatment was changed to oral metronidazole $750 \mathrm{mg}$ tid for 10 days. A convalescent titre for $E$ histolytica was performed one month after the initial test and showed a fourfold rise in titre. Follow-up ultasonography of the liver demonstrated gradual resolution of the abscess over four months.

\section{DISCUSSION}

Our patient presented with symptoms consistent with community-acquired pneumonia with concomitant pleural involvement. His clinical picture was actually precipitated by an amoebic liver abscess with extension to the right pleural cavity and lung. This case illustrates how pneumonia can be caused not only by microaspiration of organisms, inhalation of airborne organisms or seeding of the lung by blood-borne pathogens, but also by contiguous spread of a pathological process across the diaphragm.

Amebiasis caused by $E$ histolytica is endemic in tropical and subtropical countries. Cases of amebiasis seen in North America are typically caused by fecal contamination of the water supply by foreign-born individuals arriving from endemic areas. It is presumed that the patient acquired his disease in this manner via the well water in northern Ontario; this was not proven, however, because it is not known whether any subsequent tests were performed on this water source. Rec-ently, it has been determined by genetic analysis that there are distinct species of Entamoeba that are morphologically identical (1). Entamoeba dispar, a more prevalent species, is associated with an asymptomatic carrier state. In contrast, E histolytica, the pathogenic species, is associated with symptomatic infection. The cyst form of $E$ histolytica exists in contaminated water and food, and once indigested, it results in excystation in the small bowel and trophozoite infection of the colon. This produces symptomatic dysentery, the most common manifestation of amebiasis.

Liver involvement accounts for about $10 \%$ to $40 \%$ of symptomatic patients (2-4). Infection ascends from the colon, through the portal system and to the liver. Typically, patients with amoebic liver abscesses have an acute course with symptoms for fewer than 10 days. However, at times, a subacute course, with symptoms lasting many months, may prevail (4). Fewer than $30 \%$ of patients have active diarrhea at some time before presentation (1). The most common presenting symptom is pain, which may emanate from the right upper quadrant of the abdomen, the epigastrium, the right lower chest or the right shoulder (4). The pain may be dull, stabbing or pleuritic in nature. Fewer than $50 \%$ of the patients have an enlarged, painful liver. Long standing cases show weight loss, anemia, cachexia and low grade fevers.
Amoebic liver abscesses may involve the pleura, lung, pericardium or peritoneum. Pleuropulmonary involvement, particularly of the right lung, occurs in about $50 \%$ of cases (5). Pulmonary complaints include pleuritic chest pain, cough and dyspnea. The most common findings on radiographic examination of the chest are right-sided pleural effusions and elevations in the right hemidiaphragm.

The diagnosis of intestinal amebiasis is based on examination of the stool or a biopsy of mucosal tissue. Invasive disease is confirmed by finding hematophagous trophozoites of E histolytica in the stool. Microscopic examination of a wet mount from a hepatic aspirate will reveal the diagnosis in only $30 \%$ of cases. Serology is the best way to confirm the diagnosis of invasive amebiasis. An immunoglobulin $\mathrm{G}$ titre of 1:100 to $E$ histolytica-specific antigen by ELISA is considered positive. A titre greater than 1:800 has a sensitivity and specificity of greater than 95\%, especially in nonendemic areas (6).

The differential diagnosis of amoebic liver abscess includes pyogenic abscess, echinococcal cyst and hepatoma. If the diagnosis is uncertain, a fine needle aspiration under ultrasound or computed tomography guidance can be performed to rule out pyogenic disease.

The treatment of amoebic liver abscesses involves a fiveto 10-day course of oral metronidazole $750 \mathrm{mg}$ bid. The cure rate is greater than $95 \%(6)$, and the mortality rate associated with uncomplicated amoebic liver abscess is less than $1 \%$. Although aspiration of the abscess is rarely needed because there is no adequate evidence that evacuation of large lesions leads to more rapid healing, it should be entertained in the following situations: to rule out a pyogenic abscess, particularly with multiple lesions; as an adjunct to medical therapy if it appears that the patient is a therapeutic failure within three to five days; and if rupture of the abscess is believed to be imminent $(7,8)$.

Our patient was an interesting case of 'communityacquired pneumonia' as a result of pleuropulmonary involvement from an amoebic liver abscess. A high index of suspicion is necessary to make this diagnosis in nonendemic areas in combination with epidemiological risk factors, radiological findings, microbiology and serology.

\section{REFERENCES}

1. Ravdin JI. Amebiasis. Clin Infect Dis 1995;20:1453-66.

2. Maltz G, Knauer CM. Amebic liver abscess: a 15-year experience. Am J Gastroenterol 1991;86:704-10.

3. Del Campo C, Del Campo M. Thoracic complications of amebiasis. Can J Surg 1982;25:119-21.

4. Adams EB, MacLeod IN. Invasive amebiasis II. Amebic liver abscess and its complications. Medicine 1977;56:325-34.

5. Lyche KD, Jensen WA, Kirsch CM, Yenokida GG, Maltz GS, Knauer CM. Pleuropulmonary manifestations of hepatic amebiasis. West J Med 1990;153:275-8.

6. Reed SL. Amebiasis: An update. Clin Infect Dis 1992;14:385-93.

7. VanSonnenberg E, Mueller PR, Schiffman HR, et al. Intrahepatic amebic abscesses: Indications for and results of percutaneous catheter drainage. Radiology 1985;156:631-5.

8. Ralls PW, Barnes PF, Johnson MB, De Cock KM, Radin DR, Halls J. Medical treatment of hepatic amebic abscess: Rare need for percutaneous drainage. Radiology 1987;165:805-7. 


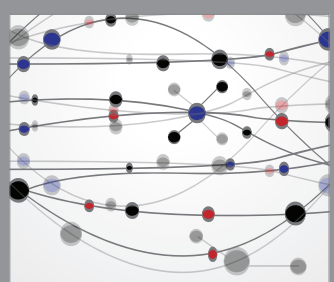

The Scientific World Journal
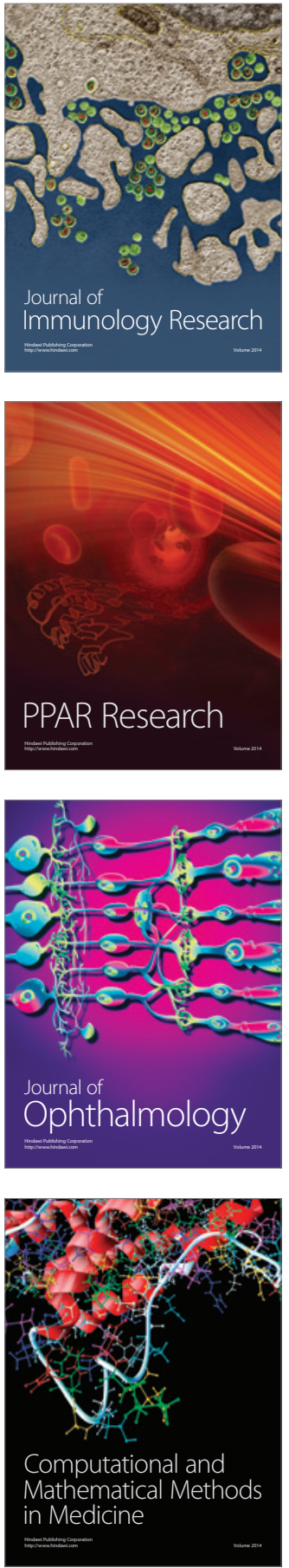

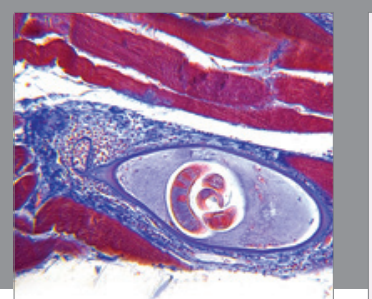

Gastroenterology Research and Practice

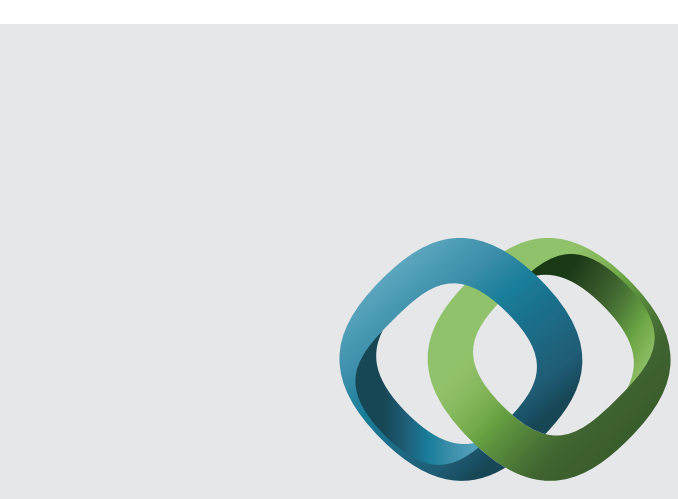

\section{Hindawi}

Submit your manuscripts at

http://www.hindawi.com
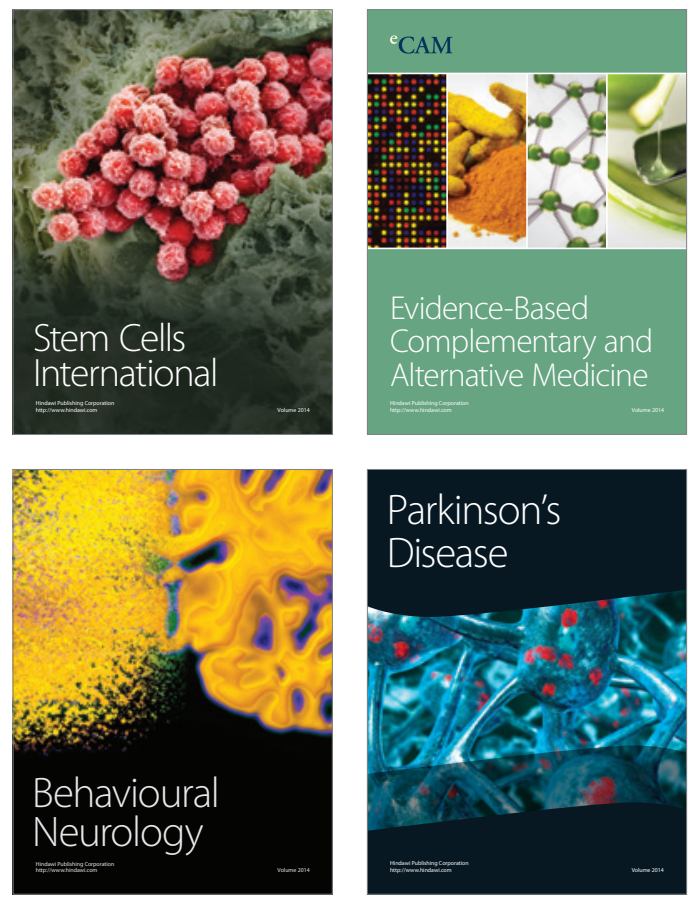
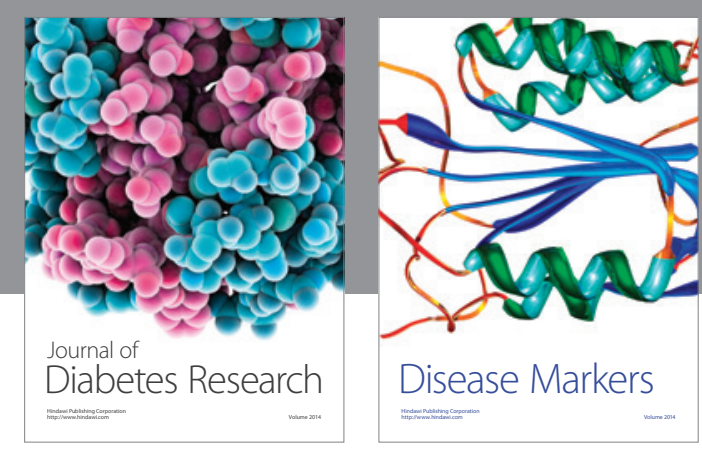

Disease Markers
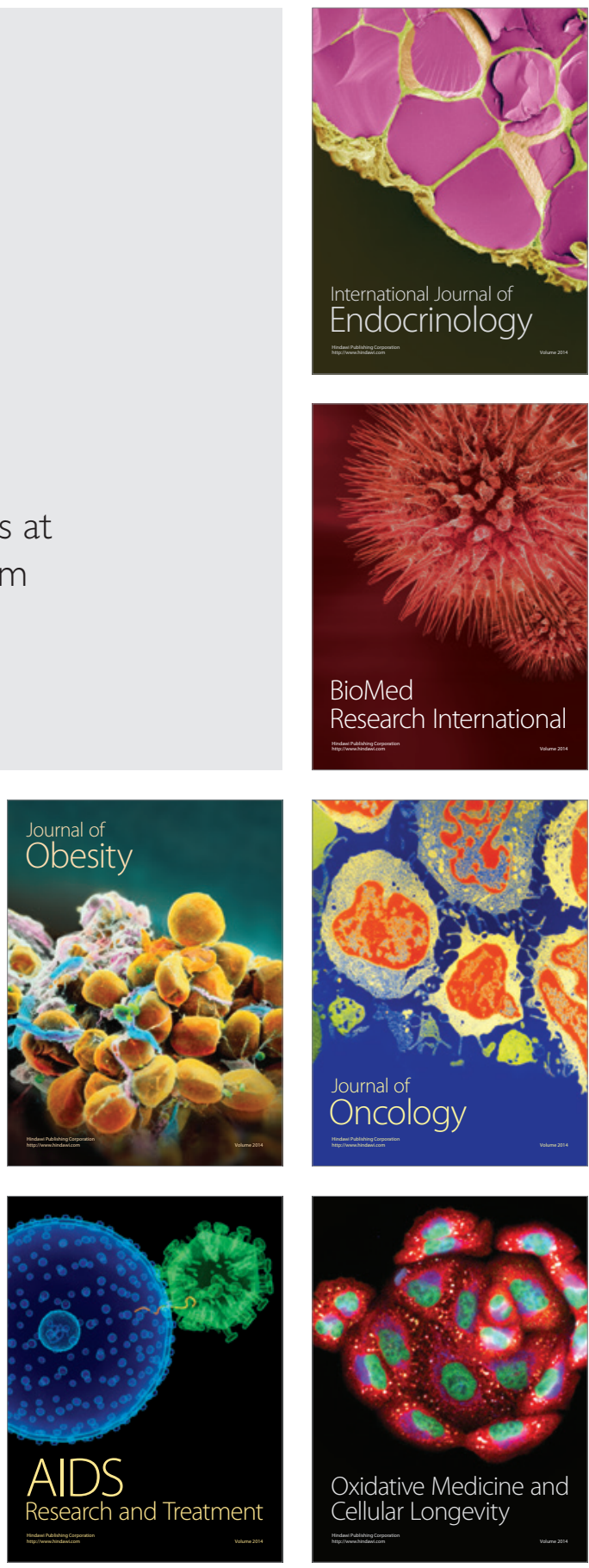